\title{
Anaesthesia for obstructive symptomatic retrosternal goiter requiring hemithyroidectomy - A case report
}

\author{
Wismans NM ${ }^{1}$ and Bouman $\mathrm{EAC}^{2 *}$ \\ ${ }^{1}$ Anaesthesiology resident, Department of Anaesthesiology and Pain Management, Maastricht University Medical Centre, Maastricht, The Netherlands \\ ${ }^{2}$ Department of Anaesthesiology and Pain Management, Maastricht University Medical Centre, Maastricht, The Netherlands
}

\begin{abstract}
Retrosternal thyroid enlargement is a challenging issue in anaesthesia because it may cause respiratory and hemodynamic compromise by obstructing the trachea or vascular structures. In this case report we present the anaesthetic management for hemithyroidectomy in an 83 year old female with stridor and dyspnoea caused by obstructing retrosternal goiter, focussing on the necessity of multidisciplinary approach and the airway strategy using venovenous extracorporeal membrane oxygenation as backup method.
\end{abstract}

\begin{abstract}
Abbreviations: CICO: cannot intubate and cannot oxygenate; VV-ECMO: venovenous extracorporeal membrane oxygenation; ECMO: extracorporeal membrane oxygenation; VA-ECMO: venoarterial extracorporeal membrane oxygenation; i.v: intravenous
\end{abstract}

\section{Introduction}

In case of retrosternal goiter the thyroid gland grows abnormally through the thoracic inlet. Patients become symptomatic depending on the structures that are compromised. They may develop dyspnoea and stridor as consequence of slow progressive growth or sudden nodular haemorrhage resulting in tracheobronchial compression. Compression of vascular structures can cause the vena cava superior syndrome with facial and upper body oedema and eventual postural collapse. Symptomatic obstructive goiter can be treated conservative with radioiodine. This therapy is relative contraindicated since there is a potential risk of transient acute thyroid enlargement with acute airway compromise. Therefore surgery is the primary choice of treatment in which approximately $2 \%$ of substernal goiters is removed by an extracervical approach [1]. Sternotomy is more likely in case of growth beyond the aortic arch, conic shape or ectopic thyroid tissue $[2,3]$.

We present the perioperative considerations and airway strategy that were made in the management of a patient with an obstructive retrosternal goiter. This case delineates the need for a multidisciplinary approach and possible challenges in anaesthesiology.

\section{Case presentation}

A 83-year-old woman with retrosternal goiter was referred to a tertiary hospital for left hemithyroidectomy after being rejected in the anaesthesia department of a secondary care centre because of serious concerns about airway management. A few years ago she was diagnosed with asymptomatic intrathoracic thyroid growth which was left untreated. Since a year she experienced a progressive stridor and dyspnoea on little exercise resulting in a maximal score of 4 in the range of metabolic equivalent of tasks. She had a medical history of hypertension, nephrectomy and transsphenoidal hypophysectomy without endocrine insufficiency. Physical examination revealed body mass index $34,05 \mathrm{~kg} / \mathrm{m}^{2}$ and normal vital signs. On airway examination no neck swelling was palpable limited by a short and broad neck. Neck mobility and thyromental distance were normal. Mouth opening was adequate with Mallampati score 1 and her remaining teeth were in good condition without impeding oral inspection. During indirect laryngoscopy performed by the otorhinolaryngologist a normal larynx mobility was seen. We found an inspiratory and expiratory stridor that was not posture dependent. Additional systemic examination showed no suggestion of superior vena cava syndrome or other abnormalities. Besides reduced renal function, blood analyses were normal with adequate thyroid function. Computed tomography in supine position demonstrated a solid, cystic enlargement of the left thyroid lobule extending into the thoracic inlet just above the aortic arch (Figure 1). The mass effect resulted in serious tracheal deviation to the right and distal tracheal compression. The narrowest tracheal lumen diameter was $3.7 \mathrm{~mm}$ in the transverse plane and was positioned $4.5 \mathrm{~cm}$ cranial to the carina. Beyond this point the airway diameter was gradually widening. Although the vasculature on the left side was closely involved, none of the remaining surrounding structures were compromised.

After multidisciplinary discussion emphasizing a difficult airway strategy concerning respiratory deterioration after induction, the patient was accepted for surgery in a specialized team consisting of a cardiovascular anaesthesiologist, general surgeon specialized in thyroid surgery, cardiothoracic surgeon and perfusionist. The main concern was this distal airway obstruction might cause problems achieving successful endotracheal intubation and rescue transtracheal access during general anaesthesia. The primary plan A was to secure the airway under optimal circumstance with conventional direct laryngoscopy and

${ }^{\star}$ Correspondence to: Bouman EAC, Department of Anaesthesiology and Pain Management, P. Debyelaan 256202 AZ Maastricht, the Netherlands, E-mail: esther.bouman@mumc.nl

Key words: obstructive goiter, airway management, thyroidectomy, anaesthesia

Received: September 11, 2019; Accepted: September 20, 2019; Published: September 23, 2019 


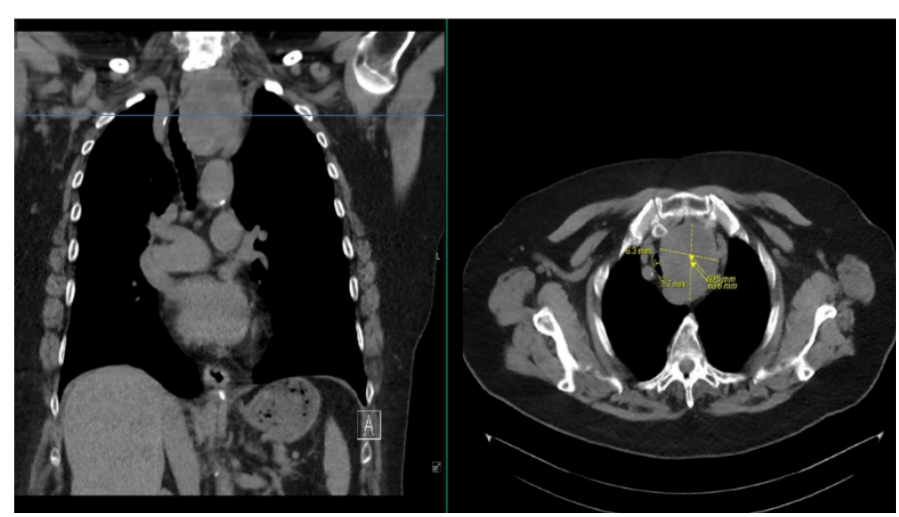

Figure 1. Computed tomography of neck and thorax showing a large substernal goiter reaching the aortic arch with tracheal deviation to the right (frontal plane on the left) and tracheal compression (transverse plane on the right). The blue line on the left corresponds with the narrowest trachea diameter on the right measuring $3.7 \mathrm{~mm}$ in the transverse plane

placement of a cuffed endotracheal tube after intravenous induction with adequate muscle relaxation. The difficult airway trolley was standby including a fiberscope and different endotracheal tube sizes. If the tube would not pass below the vocal cords plan B would be to use a microlaryngeal endotracheal tube with a smaller internal diameter. A maximum of 3 attempts was set to minimize desaturation and damage. The backup plan $\mathrm{C}$ in a cannot intubate and cannot oxygenate (CICO) situation was to provide full respiratory support with venovenous extracorporeal membrane oxygenation (VV-ECMO). Therefore the cardiothoracic surgeon and perfusionist were in the surgery room during the induction with a primed ECMO circuit and cannulas. After securing the airway the cardiothoracic surgeon was still standby in case of need for sternotomy.

Standard monitoring was applied including SpO2, ECG and BIS. In awake condition $20 \mathrm{G}$ venous access was performed in the right arm. An arterial line was inserted in the left radial artery. The right jugular vein and femoral vein were punctured under local anaesthesia leaving behind an 6 French Terumo Radifocus ${ }^{\circ}$ introducer sheath. In case of CICO this introducer would be used as cannulation site for VV-ECMO after placing a wire using the Seldinger technique and predilating. The jugular introducer was covered sterile so that it would not interfere with the surgery field. Before induction percutaneous saturation was $96 \%$. After preoxygenation with a continuous positive airway pressure of $10 \mathrm{~cm} \mathrm{H}_{2} \mathrm{O}$ PEEP the end tidal oxygen concentration was above $90 \%$. The patient was intravenous induced with midazolam $3 \mathrm{mg}$, sufentanil $15 \mathrm{mcg}$, propofol $120 \mathrm{mg}$ and rocuronium $0.6 \mathrm{mg} / \mathrm{kg}$. Anaesthesia was conducted with total intravenous anaesthesia using propofol $6-8 \mathrm{mg} /$ $\mathrm{kg} / \mathrm{u}$ and remifentanil $0,2-0,4 \mathrm{mcg} / \mathrm{kg} / \mathrm{min}$. Dexamethason $4 \mathrm{mg}$ was given intravenous (i.v.) against swelling, nausea and vomiting. After induction mask ventilation was easy without the need for high pressures. Direct laryngoscopy using the Mackintosh blade size 3 revealed a Cormack and Lehane grade 1 laryngoscopy. A cuffed endotracheal tube with $6.5 \mathrm{~mm}$ internal diameter was placed between the vocal cords without any resistance. Positive pressure ventilation was performed without any sign of high airway pressure or airway obstruction. SpO2 remained above $98 \%$ and end tidal $\mathrm{CO}_{2}$ was between 4.0-4.5 $\mathrm{kPa}$. Inspection of the trachea using a flexible bronchoscope placed through the endotracheal tube showed no abnormalities distal from the end of the tube.

The left thyroid lobule was easily removed by the surgeon through an cervical incision with the neck in extension without relevant blood loss. The recurrent nerve was monitored visually by the surgeon and was easily identified without damage. The parathyroid glands were also saved. Before extubation the surgeon palpated the trachea and concluded that the rings were patent without any sign of weakness. Under deep anaesthesia the endoluminal side of trachea was inspected again using the flexible bronchoscope. Retracting the endotracheal tube with deflated cuff just beneath the vocal cords revealed a small red spot in the 6 oclock position at the trachealis muscle. This was labelled as an superficial intubation lesion. No airway collapse was seen. Direct laryngoscopy showed no laryngeal swelling and symmetric vocal cords. The team stated that the patient could be safely extubated since there was no sign of tracheomalacia, vocal cord paralysis or swelling under anaesthesia. If there would be any sign of respiratory distress, desaturation or stridor plan A was to anaesthetize the patient including full muscle relaxation and perform reintubation the same as during induction. Plan B was to switch to VV-ECMO. In case of severe tracheomalacia the pulmonologist was standby to place an endotracheal stent.

The patient was positioned upright in bed under deep anaesthesia. Residual muscle relaxation was excluded with neuromonitoring. For postoperative analgesia the patient received $1 \mathrm{~g}$ i.v. acetaminophen and $4 \mathrm{mg}$ piritramide i.v., a synthetic opioid with comparable pharmacological profile with morphine. Ondansetron $4 \mathrm{mg}$ was given i.v. against nausea and vomiting. I.v. anaesthetics were stopped after preoxygenation with a $100 \%$ fraction of inspired oxygen. The patient woke up breathing spontaneously through the endotracheal tube without support. There was no coughing or squeezing upon extubation. After extubation there were nog signs of hoarseness, stridor, external swelling or respiratory failure. She was transported to the Intensive Care department with $2 \mathrm{~L}$ oxygen through a nasal cannula. Her vital signs remained stable without support and immediately she noticed normal speech, absence of the stridor and improvement in breathing. After six hours of observation the venous introducer sheaths were removed and the patient was transported to surgical ward.

\section{Discussion}

Anaesthesia for patients with obstructive retrosternal mass remains challenging during the entire perioperative period as there is a potential risk for total obstruction of the distal trachea after induction and for tracheomalacia after extubation.

The results of fourth National Audit Project (NAP4) of the Royal College of Anaesthetists and The Difficult Airway Society (DAS) in 2011 created awareness of the high risk for airway complications in head and neck surgery since $40 \%$ of all reported cases were in association with acute or chronic disease process in the head, neck or trachea [4]. The actual airway strategy in the anticipated difficult airway must be based on imaging, the level of airway obstruction, its severity and the nature of the abnormality. Most of the time multinodular goiter is a benign pathology without invasion of the trachea. The resulting series of plans should be agreed by an experienced team including the anaesthetist, surgeon and others involved in the strategy. The airway strategy must accept it may fail with a clear rescue plan that does not assume the patient will wake because this does not seem to be a realistic option. Hung, et al. found that airway management is 'context sensitive' since environment and expert opinion are essential besides these patient factors [5]. In case of failure of one plan the major paradigm should be to achieve oxygenation and ventilation over tracheal intubation [5].

Consensus in difficult airway management between anaesthetists can be difficult. This is well delineated in the work of Cook, et al. [6]. A group of international experts in anaesthetic airway management was asked about their strategy in case of severe trachea compression 
secondary to a retrosternal thyroid mass. Opinions differed markedly ranging from performing the awake fiberoptic intubation, conventional intubation after i.v. induction with muscle relaxation or after inhalation induction, to jet ventilation over an airway exchange catheter under sedation. Backup plans were more consistent varying between rigid bronchoscopy and cardiopulmonary bypass. A similar discrepancy was seen in extubation plans.

In this case we first discussed the airway strategy with an experienced multidisciplinary team and concluded that if any airway problem would occur it would be a subglottic, distal obstruction. Therefore plan A was a regular i.v. induction with adequate muscle relaxation because in our opinion this provides the best intubation condition. In theory muscle relaxation and positive pressure ventilation can cause progressive respiratory collapse because the decrease in functional residual capacity and reduced transpleural pressure [7]. Many airway algorithms state the ideal situation in which the patient is awake with spontaneous breathing and preserved airway reflexes. Nevertheless awake fiberoptic intubation remains controversial because of the introduction of the flexible fiberscope can cause complete airway obstruction or laryngospasm with acute respiratory failure. Other reasons for failure of this technique are poor skills, apnoea during concomitant sedation, unrecognizable anatomy, contamination and aspiration, difficulties in railroading the tube or inadequate timing of induction [8]. Although inhalation induction with a volatile anaesthetic also preserves spontaneous breathing and it creates less sufficient breathing, a long time until adequate depth of anaesthesia risking laryngoor bronchospasm [9]. Besides, volatile agents also induce muscle relaxation like intravenous medication and relaxants. Therefore we agreed that these options were less safe than i.v. induction in this specific case.

As rescue option we did not consider supraglottic airway devices since the obstruction was expected below the glottis. Likewise emergency availability of the cricothyroid membrane and upper trachea did not seem meaningful. Apart from the distal obstruction, front neck access could be difficult because of changed anatomy due to the tracheal deviation and high bleeding risk since the thyroid gland is well vascularized and in close relationship with the trachea.

In our case the intubation succeeded with a standard endotracheal tube. When critically reviewing this choice it would be more logical to use the microlaryngeal tube since this provides a smaller diameter without shortening. This equipment increases the potency of successful intubation because it fits easier beyond the narrowing. We could also have chosen for a more flexible armoured tube, but we deliberately did not use this since there is a potential risk for permanent obstruction in case of kinking.

Literature regarding airway management in obstructive retrosternal goiter is limited to case reports, case series and retrospective studies. Dempsey, et al. reviewed the anaesthetic management for thyroidectomy in patients with retrosternal goiter extending to the aortic arch [10]. The study was performed in a tertiary centre with a dedicated head and neck surgery team. 19 out of 573 thyroidectomies were performed because of retrosternal growth with a mean trachea diameter of $9.6 \mathrm{~mm}$ and $25-70 \%$ endoluminal reduction. In 95 percent of the cases thyroid enlargement was benign and multinodular. One patient had an unsuccessful inhalation induction with emergency tracheostomy. One patient had successful awake fiberoptic intubation. The other patients underwent successful intubation after i.v. induction with neuromuscular blockade and direct laryngoscopy. Even in patients with inspiratory stridor and low tracheal diameters tube sizes with internal diameter from $7.5 \mathrm{~mm}$ were used without any problems. The study concluded that for patients with benign retrosternal goiter, i.v. induction and conventional direct laryngoscopy is a safe technique in experienced hands in a specialized centre.

The use of extracorporeal membrane oxygenation in the anticipated difficult airway has been studied in retrospect by Malpas, et al. [11]. Cardiopulmonary bypass, VV-ECMO or venoarterial ECMO (VA-ECMO) were used a priori in 45 patients with airway obstruction for head and neck surgery or airway surgery in several tertiary centres. There were no significant complications or mortality reported. This study is limited and possibly biased by the retrospective design and absence of detailed prescription of the individual cases. Kim, et al. described the feasibility of ECMO in different airway problems [12]. In three cases emergency ECMO was used in response to respiratory arrest of which two patients were diagnosed brain death and died because of delayed ECMO insertion. There is no literature that regards the same design as our case, like switching toECMO with precannulation after placement ofi.v.introducer heaths before induction of anaesthesia. Potential benefits of VV-ECMO in difficult airway management are the possibility to perform surgery in the airway without the need for a endotracheal tube for respiration. ECMO has less bleeding complications comparing to cardiopulmonary bypass. VV-ECMO can be used without heparinization for a few hours when a heparin coated circuit is used. Eventually VA-ECMO can be used if there is also hemodynamic compromise. Possible complications like bleeding or distal limb ischemia should be kept in mind. When the supine position is not possible because of postural compression or in the vena cava superior syndrome, cannulation should both be performed in the femoral vessels.

Our team estimated they would have enough time to provide respiratory support with the VV-ECMO in the CICO situation since the team worked together in a well experienced setting. However, in case of CICO we could have tried to place an airway extubation catheter or small rigid bronchoscope across the tracheal narrowing with attachment of the Ventrain ${ }^{\circledR}$ device as an intermediate step during preparation for respiratory support with VV-ECMO. This seems to be attracting because, in contrast to jet ventilation, the underlying mechanism facilitates active inspiration and expiration based on gas entrainment with high speed flow of oxygen through the Ventrain ${ }^{\circ}$ device by reversing flow directions after releasing the inspiration or expiration switch. This prevents barotrauma, hyperinflation and secondary hemodynamic compromise. De Wolf, et al. showed that Ventrain ${ }^{\circ}$ provides efficient and safe reoxygenation and ventilation when used through a long, small bore airway exchange catheter in a porcine model with obstructed airway [13]. In experienced hands this side step could have been used to overcome the time from apnoea until full VV-ECMO support is achieved.

Thyroidectomy is also associated with a potential risk of developing a difficult airway in the postoperative setting because of serious complications like damage of the recurrent laryngeal nerve, bleeding, oedema and tracheomalacia. Therefor after thyroidectomy an 'at risk' extubation algoritm must be kept in mind and discussed in a multidisciplinary approach conform the intubation strategy [14]. Our highest concern after extubation was the occurrence of tracheomalacia and secondary dynamic airway collapse during expiration. Studies in the western population are not consistent about the incidence of tracheomalacia caused by varying underlying pathology, different definitions and publication bias $[3,6,10]$. If tracheomalacia occurs, less invasive therapy like prolonged positive airway pressure or transient 
tracheostomy can be performed. Depending on the severity an airway stent or tracheal surgery might be indicated.

In our case it could have been safe to leave an airway exchange catheter after extubation since a new team was responsible for the postoperative airway management in the Intensive Care department. In case of an postoperative airway emergency this catheter could have functioned as access to railroad the endotracheal tube during laryngoscopy or as bridge to VV-ECMO using the Ventrain ${ }^{\circledR}$ device as mentioned before.

\section{Conclusion}

Airway management in the obstructive retrosternal goiter should be discussed in an experienced multidisciplinary team regarding the whole perioperative period. The definitive strategy is context sensitive in which therapies like extracorporeal membrane oxygenation could be a safe option when considered in advance.

\section{Acknowledgments}

The following persons, were all involved in the actual patient management:

1. B.W. Sauren, MD, cardiovascular anaesthesiologist, Maastricht University Medical Centre, Maastricht, The Netherlands.

2. N.D. Bouvy, MD, $\mathrm{PhD}$ surgeon, Maastricht University Medical Centre, Maastricht, The Netherlands.

3. Y.M. Ganushchak, MD, $\mathrm{PhD}$, perfusionist, Maastricht University Medical Centre, Maastricht, The Netherlands.

\section{Funding information}

Not applicable.

\section{Competing interest}

There are no competing interests.

\section{References}

1. White ML, Doherty GM, Gauger PG (2008) Evidence-Based Surgical Management of Substernal Goiter. World J Surg 32: 1285-1300.

2. Huins CT, Georgalas C, Mehrzad H, Tolley NS (2008) A new classification system for retrosternal goiter based on a systematic review of its complications and management. Int J Surg 6: 71-76.

3. Simo R, Nixon IJ, Vander Poorten V, Quer M, Shaha AR, et al. (2019) Surgical management of intrathoracic goitres. Eur Arch Oto-Rhino-L 276: 305-314.

4. Patel, Pearce, Pracy (2011) NAP4. Chapter 18 Head and neck pathology pp. 143-154.

5. Hung O, Murphy (2010) Context-Sensitive Airway Management. Anesth Analg 110 982-983. [Crossref]

6. Cook TM, Morgan PJ, Hersch PE (2011) Equal and opposite expert opinion. Airway obstruction caused by a retrosternal thyroid mass: management and prospective international expert opinion. Anaesthesia 66: 828-836.

7. Blank RS, de Souza DG (2011) Anesthetic management of patients with an anterior mediastinal mass: Continuing Professional Development. Can J Anesth 58: 853-867.

8. Popat (2011) NAP4. Chapter 14: fiberoptic intubation: uses and omissions pp. 114-120

9. Dempsey GA, Snell JA, Coathup R, Jones TM (2013) Anaesthesia for massive retrosternal thyroidectomy in a tertiary referral centre. Brit J Anaesth 111: 594-599.

10. Malpas G, Hung O, Gilchrist A, Wong C, Kent B, et al. (2018) The use of extracorporeal membrane oxygenation in the anticipated difficult airway: a case report and systematic review. Can J Anesth 65: 685-697.

11. Wan Kim C, Hyung Kim D, Soo Son B, Su Cho J, Dae Kim Y, et al. (2015) The Feasibility of Extracorporeal Membrane Oxygenation in the Variant Airway Problems. Ann Thorac Cardiovasc Surg 21: 517-522. [Crossref]

12. De Wolf MWP, Gottschall R, Preussler NP, Paxian M, Enk D (2017) Emergency ventilation with the Ventrain ${ }^{\circledR}$ through an airway exchange catheter in a porcine model of complete upper airway obstruction. Can J Anesth 64: 37-44.

13. https://das.uk.com/content/das-extubation-guidelines

14. Findlay JM, Sadler GP, Bridge H, Mihai R (2011) Post-thyroidectomy tracheomalacia: minimal risk despite significant tracheal compression. Brit J Anaesth 106: 903-906.

Copyright: (C2019 Wismans NM. This is an open-access article distributed under the terms of the Creative Commons Attribution License, which permits unrestricted use, distribution, and reproduction in any medium, provided the original author and source are credited. 